\title{
Self-actuated Polymeric Valve for Autonomous Sensing and Mixing
}

\author{
Häfliger, Daniel; Marie, Rodolphe Charly Willy; Boisen, Anja
}

\section{Published in:}

The 13th International Conference on Solid-State Sensors, Actuators and Microsystems, 2005. Digest of Technical Papers. TRANSDUCERS '05.

Link to article, DOI:

10.1109/SENSOR.2005.1497385

Publication date:

2005

Document Version

Publisher's PDF, also known as Version of record

Link back to DTU Orbit

Citation (APA):

Häfliger, D., Marie, R. C. W., \& Boisen, A. (2005). Self-actuated Polymeric Valve for Autonomous Sensing and Mixing. In The 13th International Conference on Solid-State Sensors, Actuators and Microsystems, 2005. Digest of Technical Papers. TRANSDUCERS '05. (Vol. 2, pp. 1569-1572). IEEE. https://doi.org/10.1109/SENSOR.2005.1497385

\section{General rights}

Copyright and moral rights for the publications made accessible in the public portal are retained by the authors and/or other copyright owners and it is a condition of accessing publications that users recognise and abide by the legal requirements associated with these rights.

- Users may download and print one copy of any publication from the public portal for the purpose of private study or research.

- You may not further distribute the material or use it for any profit-making activity or commercial gain

- You may freely distribute the URL identifying the publication in the public portal 


\title{
SELF-ACTUATED POLYMERIC VALVE FOR AUTONOMOUS SENSING AND MIXING
}

\author{
Daniel Haefliger, Rodolphe Marie and Anja Boisen \\ $\mathrm{MIC}$ - Department of Micro and Nanotechnology, Technical University of Denmark \\ DTU-bldg. 345 east, DK-2800 Kongens Lyngby, Denmark \\ e-mail: dh@mic.dtu.dk
}

\begin{abstract}
We present an autonomously operated microvalve array for chemical sensing and mixing, which gains the actuation energy from a chemical reaction on the valve structure. An $8-\mu m$-thick flapper valve made in SU-8 is coated with stress-loaded $\mathrm{Al}$ on one side and $\mathrm{Ti}$ on the other side. The metal films keep the flapper in a flat, stress-balanced closed position. Upon contact with an analyte composed of a $\mathrm{NaOH}$ solution the $\mathrm{Al}$ film is etched from the valve surface unbalancing the surface stress and bending the flapper. A deflection of up to $45 \mu \mathrm{m}$ is observed allowing for effective release of a green marker from a reservoir. Calculations reveal that valve operation with stress originating from biochemical processes will require considerable enhancement of the actuation efficiency.
\end{abstract}

Keywords: valve, self-actuation, surface stress, polymer, microfluidic system

\section{INTRODUCTION}

There is an increasing need in autonomously working, compact and portable point-of-care microdevices for diagnostics and drug delivery [1]. Among the tasks of such devices belong independent long-term monitoring and dispensing in e.g. implants. Wide application of autonomous microsystems, however, is currently still hindered by the lack of miniature, integrated power sources. Selfactuation and -regulation circumvents this need for any external power supply. It allows for a very compact design and long device lifetime. Some self-regulating microfluidic systems developed recently use hydrogels to regulate valves [2]. The actuation is achieved by $\mathrm{pH}$-sensitive swelling of the gel. Here we present an alternative actuation principle employing surface-stress-induced bending of micromechanical components to operate a valve. The micromechanical motion is provoked by energy released in a chemical reaction on the component's surface. Such entropically induced motion is routinely used for cantilever-based biosensors measuring e.g. DNA hybridization $[3,4]$ or antibody-antigen binding, but has not yet found application in stimuli-responsive mictofluidic actuators.

The valve structure presented here can be used for autonomous mixing of liquids or as a self-operating sensor read-out for diagnostics. The system is fabricated completely in polymers. Unlike the traditionally employed silicon, polymers exhibit high biocompatibility. The fabrication of a prototype system and proof-of- principle of the autonomous valve actuation is demonstrated.

\section{PRINCIPLE}

The general layout and working principle of an array of self-actuated microvalves is shown in Fig. 1. The valves consist of square flappers suspended on one side and separating two microfluidic systems. The flappers are actuated by energy released from a chenical reaction between one of the liquids introduced in the microfluidic systems and the valve surface. For this reason, the valves are functionalized with a specific receptor substance, which induces a change in surface stress on the flapper upon reaction with the conesponding liquid. This change in surface stress provokes a micromechanical bending of the flapper and opens the valve. The valve releases the solution of the neighbouring microfluidic system into the reacting liquid, where it can be used for a chemical process or as a marker. If the marker is composed of coloured ink it can act as an autonomous read-out system for a diagnostic array. Each valve can be functionalized with a different receptor enabling parallel screening of multiple substances. By optically detecting the colour change in the microfluidic system downstream of the valve array the presence of the ink in one channel identifies a certain chemical process, which occurred on the respective valve.

\section{FABRICATION}

The feasibility of surface-stress-driven microdevices is demonstrated by fabricating a metal-coated valve array

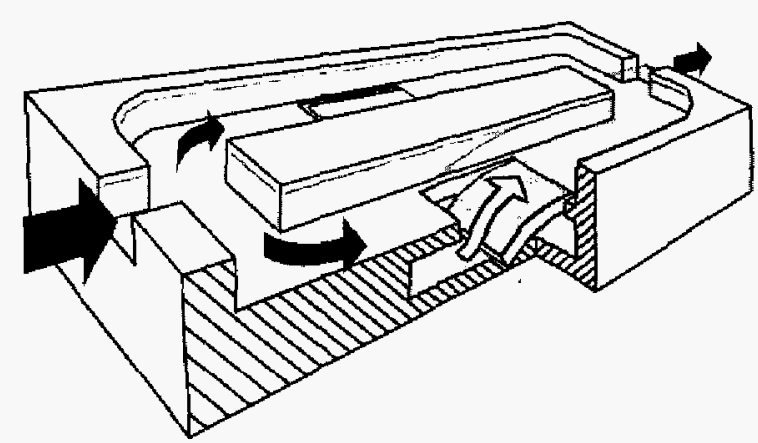

Fig. 1. Principle of microvalve array

\section{TRANSDUCERS'05}

The 13th International Conference on Solid-State Sensors, Actuators and Microsystems, Scoul, Korea, June 5-9, 2005 
(a)

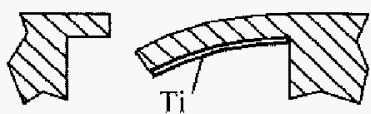

(b)

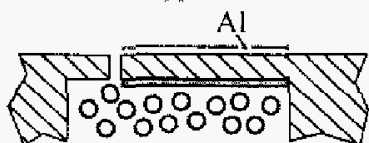

(c)

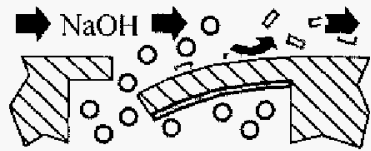

Fig. 2. Valve actuation scheme. The images show a schematic cross-section through the valve. (a) Ti coating of the bottom side of the flapper. (b) Al coating of the top side and filling of the reservoir below the valve with a marker solution. (c) Etching of the Al releases the marker.

which reacts with acids and lyes. The actuation employs elastic deformation of the valve flapper provoked by stress in the evaporation-deposited metal coating. This stress commonly arises during the deposition of the metal film. It can either be caused extrinsically by a mismatch in the thermal coefficient of expansion of the metal film and the substrate or intrinsically during the film nucleation [5]. Since the metal deposition here is performed at room temperature thermal stress can be neglected. The stress in the metal film is dominated by intrinsic sources such as material defects. For normal temperatures as here the stress in the metal film is typically tensile.

A fully self-actuated valve is achieved following the procedure outlined in Fig. 2. In our experiments the valve flapper is first coated with a thin Ti layer on the bottom side as shown in Fig. 2a. The Ti bends the flapper down towards the coated surface due to the intrinsic tensile stress. This deformation creates a gap between the flapper and the channel, which corresponds to a valve in an opened state. The valve is closed by evaporating an $\mathrm{Al}$ film on the top surface to balance the stress induced by the $\mathrm{Ti}$ layer (Fig. 2b). The deposited Al film exhibits a

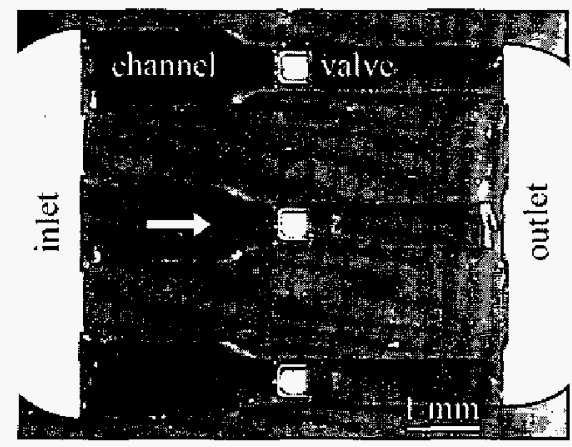

Fig. 3. Optical light microscope image of the valve array fabricated in SU-8. (a)
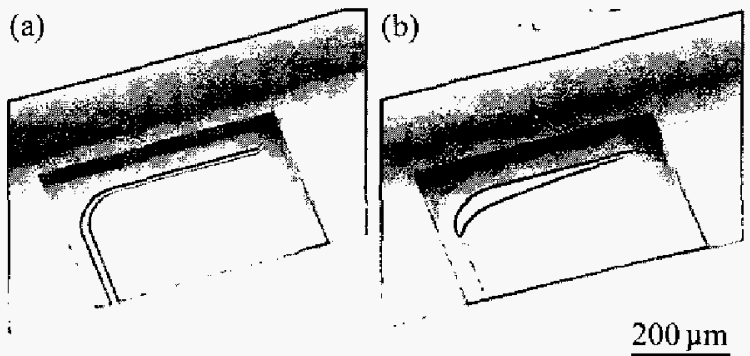

Fig. 4. Scanning electron microscope images of a fabricated valve in SU-8. (a) Closed, balanced valve as shown schematically in Fig. 2b. (b) Open valve after testing as illustrated in Fig. $2 \mathrm{c}$.

counteracting intrinsic tensile stress, which flattens the flapper and closes the gap. In this state, the flapper is ready for implementation. Upon selective etch of the $\mathrm{Al}$ film by, e.g., an $\mathrm{NaOH}$ solution as shown in Fig. 2c the tensile stress on the top of the valve is released bending the flapper back to the initial open position as depicted in Fig. 2a. By this, the valve releases a solution stored in the compartment below the flapper into the channel above.

We fabricated the valve structure in SU-8, an epoxybased negative photoresist. This UV-sensitive resin shows excellent three-dimensional fabrication properties [6] which allowed us to integrate the valve array directly into one of the two adjacent microfluidic systems. Fig. 3 shows a top view of an SU-8 chip containing an array of three valves fed by individual microfluidic channels. The valves and channels were fabricated by stacking and structuring several layers of SU-8 on a fluorocarboncoated Si wafer. The SU-8 was structured using standard UV lithography. Soft- and post-exposure baking of the resin was performed at $40^{\circ} \mathrm{C}$ to reduce thermally induced stress in the microstructure. After the microfabrication of the SU-8 was completed the individual chips were simply peeled off the Si substrate [7]

The metal films were evaporation-deposited (SCM 600 e-beam evaporator, Alcatel, France) onto the flapper after the release of the chip by the use of shadow masks. Fig. 4a shows a closed SU-8 flapper valve balanced by $160 \mathrm{~nm}$ Ti on the bottom sice (deposition rate: $10 \mathrm{~A} / \mathrm{s}$ ) and 400 $\mathrm{nm} \mathrm{Al}$ on the top side (deposition rate $2 \AA / \mathrm{s}$ ) as illustrated schematically in Fig. $2 \mathrm{~b}$. The flapper measures $400 \mu \mathrm{m} \times$ $400 \mu \mathrm{m}$ and is $8 \mu \mathrm{m}$ thick. Proper levelling of the flapper to the channel floor resulted in a minimum residual gap between the two of about $4 \mu \mathrm{m}$.

The completed valve array chip was mounted on a test rig illustrated in Fig. 5. The inlet and outlet feeds for the microfluidic network in the SU-8 chip were fabricated in a 10-mm-thick polymethylmetacrylate (PMMA) support by mechanical drilling. The feeds were equipped with threads to connect the system via PFTE tubing to a pump and reservoirs for metal etchants and water. The same PMMA support also hosted the second microfluidic sys- 


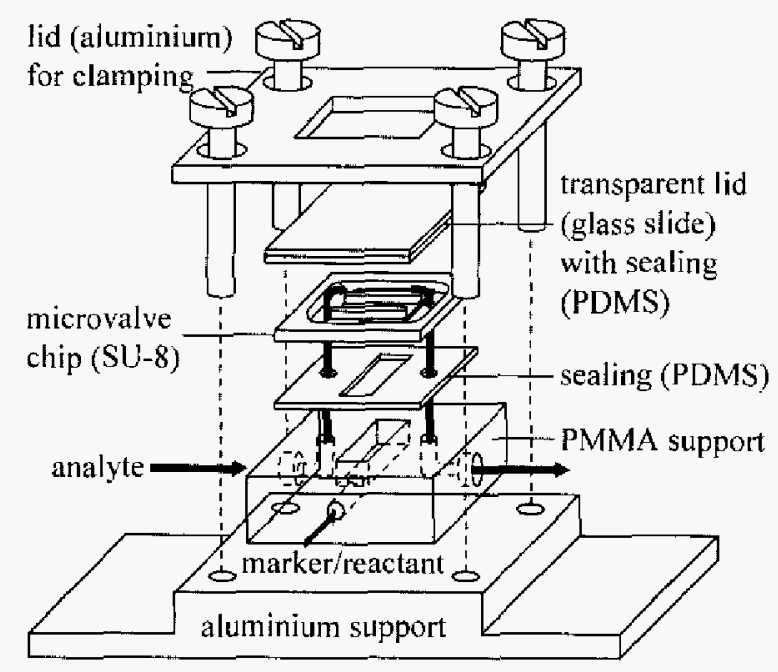

Fig. 5. Test setup for the microvalve array.

tem comprised of a reservoir with inlet and outlet holes. The reservoir supplied the complete valve array and was fabricated by infrared laser drilling [8]. The top of the valve array was covered by a glass lid with a thin layer of flexible polydimethylsiloxane (PDMS) for sealing. Another infrared-laser-machined PDMS sheet was inserted as a sealing between the $\mathrm{SU}-8$ valve chip and the PMMA support. The individual parts were clamped together by an aluminium lid screwed onto an aluminium base part. This construction allows for unobstructed observation of the valve array from above using an optical microscope.

\section{RESULTS}

To test the valve performance the fabricated array was used as a diagnostic chip to detect basic solutions. For this, the reservoir in the PMMA support below the valve array was filled with a green, water-soluble marker (nutrition colour, Ekström, Omskakas, Sweden). The microfluidic network in the SU-8 above the valves was first filled with water by a syringe pump (PHD 2000, Harvard Apparatus, Holliston, MA, USA) and rinsed for about $5 \mathrm{~min}$. Subsequently, an analyte composed of $0.5 \mathrm{M} \mathrm{NaOH}$ was introduced. The velocity of the solution over the valve amounted to about $5 \mathrm{~mm} / \mathrm{s}$. Fig. 6 shows the effective release of the green marker upon contact of the valve surface with the $\mathrm{NaOH}$ solution. The initial leakage through the residual gap between the flapper and the channel bottom proved to be negligible. The valve depicted in Fig. 6 was imaged after the testing as shown in Fig. 4b. The downward bending of the flapper is clearly visible providing evidence that the release of the marker was indeed due to a self-actuated opening of the valve. The $\mathrm{NaOH}$ solution selcctively etched the Al film, which resulted in a decrease in the layer thickness and regularly observed delamination of the film from the SU-8 substrate. Both

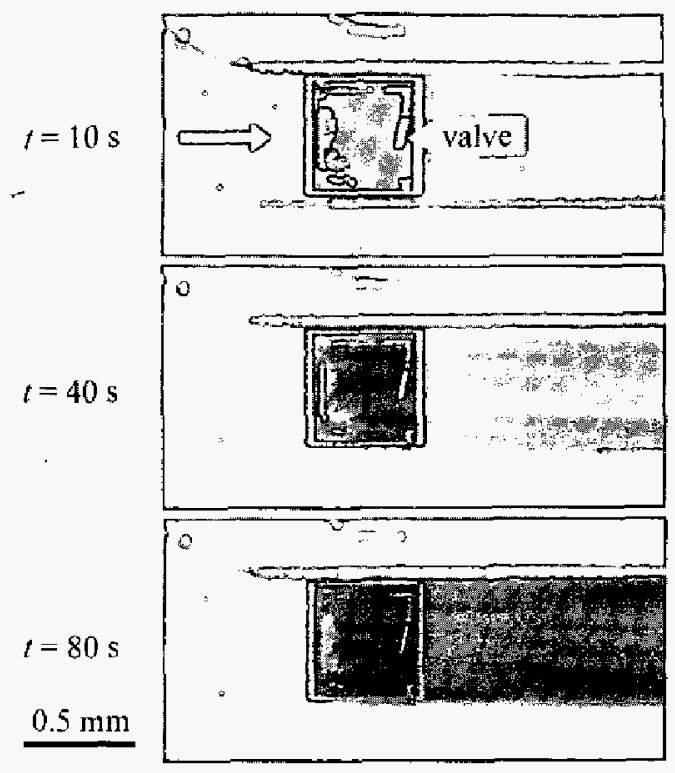

Fig. 6. Optical microscope images of a valve releasing green ink from a rescrvoir below the flapper upon introduction of a $0.5 \mathrm{M} \mathrm{NaOH}$ solution at the time $t=0$. The arrow in the top image indicates the flow direction. The Al film on the flapper is continuousiy etched and delaminating from the surface increasing the flapper deflection and thus the throughput of the ink from the reservoir. The ink is visible as the dark liquid dowustream of the valve.

mechanisms reduced the tensile stress on the top side of the flapper. The Ti layer on the bottom side remained intact during the whole experiment, pulling the flapper down as illustrated in Fig. 2c. The maximum deflection of the flapper end depicted in Fig. $4 \mathrm{~b}$ measures $45 \mu \mathrm{m}$. Here, the deflection is defined as the distance between the channel floor and the top front edge of the flapper. Valves exposed to pure water for a similar time than the duration of the whole experiment using $\mathrm{NaOH}$ showed no visible deterioration of the $\mathrm{Al}$ film. The valves remained closed providing evidence of a specific lye-induced selfactuation of the valves in contact with $\mathrm{NaOH}$.

\section{DISCUSSION}

As illustrated by Fig. 6 effective release of a marker or reactant into a microfluidic system demands for a relatively large deflection of the flapper of several tens of micrometers. The stress in the Al film driving the micromechanical motion was estimated using simple cantilever deformation theory [9]. The deflection $u$ of a cantilever is described by:

$$
\frac{d^{2} u}{d x^{2}}=-\frac{M}{\sum_{i} \hat{E}_{i} I_{i}},
$$


where $x$ is the coordinate along the flapper axis as shown in Fig. 2, $M$ the bending moment, $\hat{E}_{i}$ the individual apparent Young's modulus and $I_{i}$ the individual cross-sectional area moment of inertia of the $\mathrm{Al}, \mathrm{SU}-8$ and Ti layers of the flapper. The apparent Young's modulus for the platelike flapper here is $\hat{E}_{i}=E_{i} /\left(1-v_{i}^{2}\right)$ with $v_{i}$ the material's Poisson's ratio. The bending moment is calculated as $M=w h_{\mathrm{Al}} \sigma z_{0}$, where $w$ is the flapper width, $h_{\mathrm{Al}}$ the thickness of the Al film, $\sigma$ the film stress in the Al and $z_{0}$ the distance of the Al film from the flapper's neutral axis. Considering a flapper as shown in Fig. 4 yielding a maximum deflection of $45 \mu \mathrm{m}$ the stress in the Al film was estimated to about $400 \mathrm{MPa}$. Note that this value is approximative since the calculation neglects the influence of the flapper clamping and intrinsic stress in the SU-8 substrate originating from the fabrication process.

In the following, this stress is compared to stress induced by biomolecular films. The actuation of the valve by biochemical reactions is desirable to increase the versatility of the autonomous diagnostic array proposed here. For example, for the immobilisation of a single-stranded DNA monolayer a surface stress between $0.01-0.9 \mathrm{~N} / \mathrm{m}$ has been reported [4], corresponding to a film stress of about 1-90 MPa. The stress induced by a macromolecular film is thus up to two orders of magnitude smaller than the stress created by a several hundred nanometer thick metal film. The stress from biomolecules usually deflects a 100-200- $\mu \mathrm{m}$-long cantilever only a few hundred nanometers. As shown here the reliable operation of the valve for microfluidic systems require motions on the micrometer-scale. The actuation efficiency of structures powered by biochemical processes thus needs to be enhanced to achieve appropriate, micrometer-scaled actuation.

\section{CONCLUSION AND OUTLOOK}

We have shown proof-of-principle of a self-actuated polymeric valve by using a valve array as a diagnostic device to identify the presence of a basic solution. The actuation employs a change in the surface stress on the valve flapper induced by a chemical reaction between the analyte and the valve surface. $A$ valve array consisting of 8 - $\mu$ m-thick flappers coated with stress-loaded films of $400 \mathrm{~nm} \mathrm{Al}$ on one side and $160 \mathrm{~nm} \mathrm{Ti}$ on the other side has been fabricated in SU-8. Etching away the Al film from the flapper by a $\mathrm{NaOH}$ solution resulted in a deflection of the flapper of $45 \mu \mathrm{m}$. This micromechanical motion was sufficient to effectively release an optically detectable green ink marking the chemical reaction.

In the future, the application of such a microvalve array to more specific bio-/chemical detection is planned. A comparison between the stress induced by biomolecular layers and the metal films employed here indicates that the actuation of the valve by biochenical interactions require strategies to enhance the actuation efficiency. This will guarantee similar reliable operation as observed here.
Moreover, the pump for the analyte transport can be replaced in a future setup by capillary force driven microfluidics [10] rendering any energy source completely unnecessary. Further use of the array for targeted drug delivery can be envisioned.

\section{Acknowledgements}

The authors thank P. A. Rasmussen from Cantion A/S for helpful discussions. They further acknowledge financial support from FTP, project 2116-04-0011 on autonomous diagnostic arrays. D.H. acknowledges support through a fellowship of the Swiss National Science Foundation.

\section{References}

[1] J. T. Santini Jr, M. J. Cima, R. Langer, "A controlled-release microchip", Nature, 397, 335-338 (1999).

[2] D. J. Beebe, J. S. Moore, J. M. Bauer, Q. Yu, R. H. Liu, C. Devadoss, B. H. Jo, "Functional hydrogel structures for autonomous flow control inside microfluidic channels", Nature, 404, 588-590 (2000).

[3] J. Fritz, $\dot{M}$. K. Baller, H. P. Lang, H. Rothuizen, P. Vettiger, E. Meyer, H. J. Güntherodt, C. Gerber, J. K. Gimzewski, "Translating biomolecular recognition into nanomechanics", Science, 288, 316-318 (2000).

[4] R. Marie, H. Jensenius, J. Thaysen, C. B. Christensen, A. Boisen, "Adsorption kineties and mechanical properties of thiol-modified DNA-oligos on gold investigated by microcantilever sensors", Ultramicroscopy, 91, 29-36 (2002).

[5] M. Madou, Fundamentals of Microfabrication, pp. 219-231, CRC Press, Boca Raton, FL, USA, 1997.

[6] D. Haefliger, A. Boisen, "Contact printed masks for 3D microfabrication in negative resists", Tech. Digest IEEE MEMS 2005, pp. 556-559, Miami Beach, FL, USA, Jan. 30-Feb. 3, 2005.

[7] D. Haefliger, M. Nordström, P. A. Rasmussen, A. Boisen, "Dry release of all-polymer structures", $\mathrm{Mi}$ croelectron. Eng, published online Feb. 15, 2005.

[8] H. Klank, J. P. Kutter, O. Geschke, " $\mathrm{CO}_{2}$-laser micromachining and back-end processing for rapid production of PMMA-based microfluidic systems", $L a b$ Chip, 2, 242-246 (2002).

[9] P. A. Rasmussen, J. Thaysen, O. Hansen, S. C. Eriksen, A. Boisen, „Optimised cantilever biosensor with piezoresistive read-out", Ultramicroscopy, 97, 371376 (2003).

[10] M. Nordström, R. Marie, M. Calleja, A. Boisen, "Rendering SU-8 hydrophilic to facilitate use in micro channel fabrication", J. Micromech. Microeng., $14,1614-1617$ (2004). 